\title{
COVERINGS OF SKEW-PRODUCTS AND CROSSED PRODUCTS BY COACTIONS
}

\author{
DAVID PASK, JOHN QUIGG and AIDAN SIMS ${ }^{凶}$
}

(Received 4 June 2007; accepted 17 October 2007)

Communicated by G. A. Willis

\begin{abstract}
Consider a projective limit $G$ of finite groups $G_{n}$. Fix a compatible family $\delta^{n}$ of coactions of the $G_{n}$ on a $C^{*}$-algebra $A$. From this data we obtain a coaction $\delta$ of $G$ on $A$. We show that the coaction crossed product of $A$ by $\delta$ is isomorphic to a direct limit of the coaction crossed products of $A$ by the $\delta^{n}$. If $A=C^{*}(\Lambda)$ for some $k$-graph $\Lambda$, and if the coactions $\delta^{n}$ correspond to skew-products of $\Lambda$, then we can say more. We prove that the coaction crossed product of $C^{*}(\Lambda)$ by $\delta$ may be realized as a full corner of the $C^{*}$-algebra of a $(k+1)$-graph. We then explore connections with Yeend's topological higher-rank graphs and their $C^{*}$-algebras.
\end{abstract}

2000 Mathematics subject classification: primary 46L05; secondary 46L55.

Keywords and phrases: $C^{*}$-algebra, coaction, covering, crossed-product, graph algebra, $k$-graph.

\section{Introduction}

In this paper we investigate how certain coactions of discrete groups on $k$-graph $C^{*}$-algebras behave under inductive limits. This leads to interesting new connections between $k$-graph $C^{*}$-algebras, nonabelian duality, and Yeend's topological higher-rank graph $C^{*}$-algebras.

We consider a particularly tractable class of coactions of finite groups on $k$-graph $C^{*}$-algebras. A functor $c$ from a $k$-graph $\Lambda$ to a discrete group $G$ gives rise to two natural constructions. At the level of $k$-graphs, one may construct the skew-product $k$-graph $\Lambda \times{ }_{c} G$; and at the level of $C^{*}$-algebras, $c$ induces a coaction $\delta$ of $G$ on $C^{*}(\Lambda)$. It is a theorem of [15] that these two constructions are compatible in the sense that the $k$-graph algebra $C^{*}\left(\Lambda \times{ }_{c} G\right)$ is canonically isomorphic to the coaction crossed-product $C^{*}$-algebra $C^{*}(\Lambda) \times_{\delta} G$.

The skew-product construction is also related to discrete topology: given a regular covering map from a $k$-graph $\Gamma$ to a connected $k$-graph $\Lambda$, one obtains an isomorphism

This research was supported by the ARC.

(C) 2009 Australian Mathematical Society 1446-7887/2009 \$16.00 
of $\Gamma$ with a skew-product of $\Lambda$ by a discrete group $G$ [15, Theorem 6.11]. Further results of [15] then show how to realize the $C^{*}$-algebra of $\Gamma$ as a coaction crossed product of the $C^{*}$-algebra of $\Lambda$.

The results of [12] investigate the relationship between $C^{*}(\Lambda)$ and $C^{*}(\Gamma)$ from a different point of view. Specifically, they show how a covering $p$ of a $k$-graph $\Lambda$ by a $k$-graph $\Gamma$ induces an inclusion of $C^{*}(\Lambda)$ into $C^{*}(\Gamma)$. A sequence of compatible coverings therefore gives rise to an inductive limit of $C^{*}$-algebras. The main results of [12] show how to realize this inductive limit as a full corner in the $C^{*}$-algebra of a $(k+1)$-graph.

We can combine the ideas discussed in the preceding three paragraphs as follows. Fix a $k$-graph $\Lambda$, a projective sequence of finite groups $G_{n}$, and a sequence of functors $c_{n}: \Lambda \rightarrow G_{n}$ which are compatible with the projective structure. We obtain from this data a sequence of skew-products $\Lambda \times_{c_{n}} G_{n}$ which form a sequence of compatible coverings of $\Lambda$. By results of [12], we therefore obtain an inductive system of $k$-graph $C^{*}$-algebras $C^{*}\left(\Lambda \times_{c_{n}} G_{n}\right)$. The results of [15] show that each $C^{*}\left(\Lambda \times_{c_{n}} G_{n}\right)$ is isomorphic to a coaction crossed product $C^{*}(\Lambda) \times \delta^{n} G_{n}$. It is therefore natural to ask whether the direct limit $C^{*}$-algebra $\underset{\lim }{\longrightarrow}\left(C^{*}\left(\Lambda \times_{c_{n}} G_{n}\right)\right)$ is isomorphic to a coaction crossed product of $C^{*}(\Lambda)$ by the projective limit group $\lim G_{n}$.

After summarizing in Section 2 the background needed for our results, we answer this question in the affirmative and in greater generality in Theorem 3.1. Given a $C^{*}$-algebra $A$, a projective limit of finite groups $G_{n}$ and a compatible system of coactions of the $G_{n}$ on $A$, we show that there is an associated coaction $\delta$ of $\underset{\lim }{\longleftarrow} G_{n}$ on $A$, such that $A \times_{\delta}\left(\lim _{\longleftarrow} G_{n}\right) \cong \underset{\lim }{\longrightarrow}\left(A \times \delta^{n} G_{n}\right)$.

In Section 4, we consider the consequences of Theorem 3.1 in the original motivating context of $k$-graph $C^{*}$-algebras. We consider a $k$-graph $\Lambda$ together with functors $c_{n}: \Lambda \rightarrow G_{n}$ which are consistent with the projective limit structure on the $G_{n}$. In Theorem 4.3, we use Theorem 3.1 to deduce that $C^{*}(\Lambda) \times{ }_{\delta} G$ is isomorphic to $\lim \left(C^{*}(\Lambda) \times \delta^{n} G_{n}\right)$. Using results of [12], we realize $C^{*}(\Lambda) \times \delta G$ as a full corner in $\overrightarrow{\mathrm{a}}(k+1)$-graph algebra (Corollary 4.5). We digress in Section 5 to investigate simplicity of $C^{*}(\Lambda) \times{ }_{\delta} G$ via the results of [18].

We conclude in Section 6 with an investigation of the connection between our results and Yeend's notion of a topological $k$-graph $[20,21]$. We construct from an infinite sequence of coverings $p_{n}: \Lambda_{n+1} \rightarrow \Lambda_{n}$ of $k$-graphs a projective limit $\Lambda$ which is a topological $k$-graph. We show that the $C^{*}$-algebra $C^{*}(\Lambda)$ of this topological $k$-graph coincides with the direct limit of the $C^{*}\left(\Lambda_{n}\right)$ under the inclusions induced by the $p_{n}$. In particular, the system of cocycles $c_{n}: \Lambda \rightarrow G_{n}$ discussed in the preceding paragraph yields a cocycle $c: \Lambda \rightarrow G:=\lim \left(G_{n}, q_{n}\right)$, the skew-product $\Lambda \times{ }_{c} G$ is a topological $k$-graph, and the $C^{*}$-algebras $\overleftarrow{C^{*}}\left(\Lambda \times_{c} G\right)$ and $C^{*}(\Lambda) \times{ }_{\delta} G$ are isomorphic, generalizing the corresponding result [15, Theorem 7.1(ii)] for discrete groups.

\section{Preliminaries}

Throughout this paper, we regard $\mathbb{N}^{k}$ as a semigroup under addition with identity element 0 . We denote the canonical generators of $\mathbb{N}^{k}$ by $e_{1}, \ldots, e_{k}$. For $n \in \mathbb{N}^{k}$, 
we denote its coordinates by $n_{1}, \ldots, n_{k} \in \mathbb{N}$ so that $n=\sum_{i=1}^{k} n_{i} e_{i}$. For $m, n \in \mathbb{N}^{k}$, we write $m \leq n$ if $m_{i} \leq n_{i}$ for all $i \in\{1, \ldots, k\}$.

We will at times need to identify $\mathbb{N}^{k}$ with the subsemigroup of $\mathbb{N}^{k+1}$ consisting of elements $n$ whose last coordinate is equal to zero. For $n \in \mathbb{N}^{k}$, we write $(n, 0)$ for the corresponding element of $\mathbb{N}^{k+1}$. When convenient, we regard $\mathbb{N}^{k}$ as (the morphisms of) a category with a single object in which the composition map is the usual addition operation in $\mathbb{N}^{k}$.

2.1. $\boldsymbol{k}$-graphs Higher-rank graphs are defined in terms of categories. In this paper, given a category $\mathcal{C}$, we will identify the objects with the identity morphisms, and think of $\mathcal{C}$ as the collection of morphisms only. We will write composition in our categories by juxtaposition.

Fix an integer $k \geq 1$. A $k$-graph is a pair $(\Lambda, d)$ where $\Lambda$ is a countable category and $d: \Lambda \rightarrow \mathbb{N}^{k}$ is a functor satisfying the factorization property: whenever $\lambda \in \Lambda$ and $m, n \in \mathbb{N}^{k}$ satisfy $d(\lambda)=m+n$, there are unique $\mu, v \in \Lambda$ with $d(\mu)=m, d(v)=n$, and $\lambda=\mu \nu$. For $n \in \mathbb{N}^{k}$, we write $\Lambda^{n}$ for $d^{-1}(n)$. If $p \leq q \leq d(\lambda)$, we denote by $\lambda(p, q)$ the unique path in $\Lambda^{q-p}$ such that $\lambda=\lambda^{\prime} \lambda(p, q) \lambda^{\prime \prime}$ for some $\lambda^{\prime} \in \Lambda^{p}$ and $\lambda^{\prime \prime} \in \Lambda^{d(\lambda)-q}$.

Applying the factorization property with $m=0, n=d(\lambda)$ and with $m=d(\lambda)$, $n=0$, one shows that $\Lambda^{0}$ is precisely the set of identity morphisms in $\Lambda$. The codomain and domain maps in $\Lambda$ therefore determine maps $r, s: \Lambda \rightarrow \Lambda^{0}$. We think of $\Lambda^{0}$ as the vertices - and $\Lambda$ as the paths - in a ' $k$-dimensional directed graph'.

Given $F \subset \Lambda$ and $v \in \Lambda^{0}$, we write $v F$ for $F \cap r^{-1}(v)$ and $F v$ for $F \cap s^{-1}(v)$. We say that $\Lambda$ is row-finite if $v \Lambda^{n}$ is a finite set for all $v \in \Lambda^{0}$ and $n \in \mathbb{N}^{k}$, and we say that $\Lambda$ has no sources if $v \Lambda^{n}$ is always nonempty.

We denote by $\Omega_{k}$ the $k$-graph $\Omega_{k}:=\left\{(p, q) \in \mathbb{N}^{k} \times \mathbb{N}^{k}: p \leq q\right\}$ with $r(p, q):=$ $(p, p), s(p, q):=(q, q)$ and $d(p, q):=q-p$. As a notational convenience, we will henceforth denote $(p, p) \in \Omega_{k}^{0}$ by $p$. An infinite path in a $k$-graph $\Lambda$ is a degreepreserving functor (otherwise known as a k-graph morphism) $x: \Omega_{k} \rightarrow \Lambda$. The collection of all infinite paths is denoted $\Lambda^{\infty}$. We write $r(x)$ for $x(0)$, and think of this as the range of $x$.

For $\lambda \in \Lambda$ and $x \in s(\lambda) \Lambda^{\infty}$, there is a unique infinite path $\lambda x \in r(\lambda) \Lambda^{\infty}$ satisfying $(\lambda x)(0, p):=\lambda x(0, p-d(\lambda))$ for all $p \geq d(\lambda)$. In particular, $r(x) x=x$ for all $x \in \Lambda^{\infty}$, so we denote $\left\{x \in \Lambda^{\infty}: r(x)=v\right\}$ by $v \Lambda^{\infty}$. If $\Lambda$ has no sources, then $v \Lambda^{\infty}$ is nonempty for all $v \in \Lambda^{0}$.

The factorization property also guarantees that for $x \in \Lambda^{\infty}$ and $n \in \mathbb{N}^{k}$ there is a unique infinite path $\sigma^{n}(x) \in x(n) \Lambda^{\infty}$ such that $\sigma^{n}(x)(p, q)=x(p+n, q+n)$. We somewhat imprecisely refer to $\sigma$ as the shift map. Note that $\sigma^{d(\lambda)}(\lambda x)=x$ for all $\lambda \in \Lambda, x \in s(\lambda) \Lambda^{\infty}$, and $x=x(0, n) \sigma^{n}(x)$ for all $x \in \Lambda^{\infty}$ and $n \in \mathbb{N}^{k}$.

We say that a row-finite $k$-graph $\Lambda$ with no sources is cofinal if, for every $v \in \Lambda^{0}$ and every $x \in \Lambda^{\infty}$, there exists $n \in \mathbb{N}^{k}$ such that $v \Lambda x(n) \neq \emptyset$. Given $m \neq n \in \mathbb{N}^{k}$ and $v \in \Lambda^{0}$, we say that $\Lambda$ has local periodicity $m, n$ at $v$ if $\sigma^{m}(x)=\sigma^{n}(x)$ for 
all $x \in v \Lambda^{\infty}$. We say that $\Lambda$ has no local periodicity if, for every $m, n \in \mathbb{N}^{k}$ and every $v \in \Lambda^{0}$, we have $\sigma^{m}(x) \neq \sigma^{n}(x)$ for some $x \in v \Lambda^{\infty}$.

2.2. Skew-products Let $\Lambda$ be a $k$-graph, and let $G$ be a group. A cocycle $c: \Lambda \rightarrow G$ is a functor from $\Lambda$ to $G$ where the latter is regarded as a category with one object. That is, $c: \Lambda \rightarrow G$ satisfies $c(\mu \nu)=c(\mu) c(v)$ whenever $\mu, v$ can be composed in $\Lambda$. It follows that $c(v)=e$ for all $v \in \Lambda^{0}$, where $e \in G$ is the identity element.

Given a cocycle $c: \Lambda \rightarrow G$, we can form the skew-product $k$-graph $\Lambda \times{ }_{c} G$. We follow the conventions of [15, Section 6]. Note that these are different from those of [9, Section 5]. The paths in $\Lambda \times{ }_{c} G$ are

$$
\left(\Lambda \times_{c} G\right)^{n}:=\Lambda^{n} \times G
$$

for each $n \in \mathbb{N}^{k}$. The range and source maps $r, s: \Lambda \times{ }_{c} G \rightarrow\left(\Lambda \times_{c} G\right)^{0}$ are given by $r(\lambda, g):=(r(\lambda), c(\lambda) g)$ and $s(\lambda, g):=(s(\lambda), g)$. Composition is determined by $(\mu, c(\nu) g)(\nu, g)=(\mu \nu, g)$. It is shown in [15, Section 6] that $\Lambda \times_{c} G$ is a $k$-graph.

2.3. Coverings and $(\boldsymbol{k}+\mathbf{1})$-graphs We recall here some definitions and results from [12] regarding coverings of $k$-graphs. Given $k$-graphs $\Lambda$ and $\Gamma$, a $k$-graph morphism $\phi: \Lambda \rightarrow \Gamma$ is a functor which respects the degree maps. A covering of $k$-graphs is a triple $(\Lambda, \Gamma, p)$ where $\Lambda$ and $\Gamma$ are $k$-graphs, and $p: \Gamma \rightarrow \Lambda$ is a $k$-graph morphism which is surjective and is locally bijective in the sense that for each $v \in \Gamma^{0}$, the restrictions $\left.p\right|_{v \Gamma}: v \Gamma \rightarrow p(v) \Lambda$ and $\left.p\right|_{\Gamma v}: \Gamma v \rightarrow \Lambda p(v)$ are bijective.

REMARK 2.1. What we have called a covering of $k$-graphs is a special case of what was called a 'covering system of $k$-graphs' in [12]. In general, a covering system consists of a covering of $k$-graphs together with some extra combinatorial data. We do not need the extra generality, so we have dropped the word 'system'.

A covering $(\Lambda, \Gamma, p)$ is row-finite if $\Lambda$ (equivalently $\Gamma$ ) is row-finite, and $\left|p^{-1}(v)\right|<\infty$ for all $v \in \Lambda^{0}$. By [12, Proposition 2.6] we can associate to a row-finite covering $p: \Gamma \rightarrow \Lambda$ of $k$-graphs a row-finite $(k+1)$-graph $\Lambda \stackrel{p}{\leftarrow} \Gamma$ containing disjoint copies $\imath(\Lambda)$ and $J(\Gamma)$ of $\Lambda$ and $\Gamma$ with an edge of degree $e_{k+1}$ connecting each vertex $\jmath(v) \in J\left(\Gamma^{0}\right)$ to its image $l(p(v)) \in l\left(\Lambda^{0}\right)$.

More generally, given a sequence $\left(\Lambda_{n}, \Lambda_{n+1}, p_{n}\right)$ of row-finite coverings of $k$ graphs, [12, Corollary 2.10] shows how to build a $(k+1)$-graph $\lim \left(\Lambda_{n} ; p_{n}\right)$, which we sometimes refer to as a tower graph, containing a copy $l_{n}\left(\Lambda_{n}\right)$ of each individual $k$-graph in the sequence, and an edge of degree $e_{k+1}$ connecting each $l_{n+1}(v) \in$ $\imath_{n+1}\left(\Lambda_{n+1}^{0}\right)$ to its image $\imath_{n}\left(p_{n}(v)\right) \in \imath_{n}\left(\Lambda_{n}^{0}\right)$. The $(k+1)$-graph $\lim \left(\Lambda_{n} ; p_{n}\right)$ has no sources if the $\Lambda_{n}$ all have no sources.

Given a covering $(\Lambda, \Gamma, p),[12$, Proposition 3.2 and Theorem 3.8] show that the covering map $p: \Gamma \rightarrow \Lambda$ induces an inclusion $\iota_{p}: C^{*}(\Lambda) \rightarrow C^{*}(\Gamma)$. If $\left(\Lambda_{n}, \Lambda_{n+1}, p_{n}\right)_{n=1}^{\infty}$ is a sequence of coverings, the $(k+1)$-graph algebra $C^{*}\left(\lim \left(\Lambda_{n} ; p_{n}\right)\right)$ is Morita equivalent to the direct limit $\underset{\lim }{\longrightarrow}\left(C^{*}\left(\Lambda_{n}\right), \iota_{p_{n}}\right)$. 
2.4. Coactions and coaction crossed products Here we give some background on group coactions on $C^{*}$-algebras and coaction crossed products. For a detailed treatment of coactions and coaction crossed products, see [4, Appendix A].

Given a locally compact group $G$, we write $C^{*}(G)$ for the full group $C^{*}$-algebra of $G$. We prefer to identify $G$ with its canonical image in $M\left(C^{*}(G)\right)$, but when confusion is likely we use $s \mapsto u(s)$ for the canonical inclusion of $G$ in $M\left(C^{*}(G)\right)$. If $A$ and $B$ are $C^{*}$-algebras, then $A \otimes B$ denotes the spatial tensor product. For a group $G$, we write $\delta_{G}$ for the natural comultiplication $\delta_{G}: C^{*}(G) \rightarrow M\left(C^{*}(G) \otimes C^{*}(G)\right)$ given by the integrated form of the strictly continuous map which takes $s \in G$ to $s \otimes s \in \mathcal{U} M\left(C^{*}(G) \otimes C^{*}(G)\right)$.

As in [4, Definition A.21], a coaction of a group $G$ on a $C^{*}$-algebra $A$ is an injective homomorphism $\delta: A \rightarrow M\left(A \otimes C^{*}(G)\right)$ satisfying:

(1) the coaction identity $\left(\delta \otimes 1_{G}\right) \circ \delta=\left(1_{A} \otimes \delta_{G}\right) \circ \delta$ (as maps from $A$ to $M(A \otimes$ $\left.\left.C^{*}(G) \otimes C^{*}(G)\right)\right)$; and

(2) the nondegeneracy condition $\overline{\delta(A)\left(1_{A} \otimes C^{*}(G)\right)}=M\left(A \otimes C^{*}(G)\right)$.

As in $[7,8]$, the nondegeneracy condition (2) — rather than the weaker condition that $\delta$ be a nondegenerate homomorphism - is part of our definition of a coaction (compare with [4, Definition A.21 and Remark A.22(3)]). Since we will be dealing only with coactions of compact (and hence amenable) groups, the two conditions are equivalent in our setting in any case (see [14, Lemma 3.8]).

Let $\delta: A \rightarrow M\left(A \otimes C^{*}(G)\right)$ be a coaction of $G$ on $A$. We regard the map which takes $s \in G$ to $u(s) \in M\left(C^{*}(G)\right)$ as an element $w_{G}$ of $\mathcal{U} M\left(C_{0}(G) \otimes C^{*}(G)\right)$. Given a $C^{*}$-algebra $D$, A covariant homomorphism of $(A, G, \delta)$ into $M(D)$ is a pair $(\pi, \mu)$ of homomorphisms $\pi: A \rightarrow M(D)$ and $\mu: C_{0}(G) \rightarrow M(D)$ satisfying the covariance condition:

$$
\left(\pi \otimes \mathrm{id}_{G}\right) \circ \delta(a)=\left(\mu \otimes \operatorname{id}_{G}\right)\left(w_{G}\right)(\pi(a) \otimes 1)\left(\mu \otimes \mathrm{id}_{G}\right)\left(w_{G}\right)^{*},
$$

for all $a \in A$.

The coaction crossed product $A \rtimes_{\delta} G$ is the universal $C^{*}$-algebra generated by the image of a universal covariant representation $\left(j_{A}, j_{G}\right)$ of $(A, G, \delta)$ (see [4, Theorem A.41]).

\section{Continuity of coaction crossed products}

In this section, we prove a general result regarding the continuity of the coaction crossed-product construction. Specifically, consider a projective system of finite groups $G_{n}$ and a system of compatible coactions $\delta^{n}$ of the $G_{n}$ on a fixed $C^{*}$-algebra $A$. We show that this determines a coaction $\delta$ of the projective $\operatorname{limit} \lim G_{n}$ on $A$, and that the coaction crossed product of $A$ by $\delta$ is isomorphic to a direct limit of the coaction crossed products of $A$ by the $\delta^{n}$. 
The application we have in mind is when $A=C^{*}(\Lambda)$ is a $k$-graph algebra, and the $\delta^{n}$ arise from a system of skew-products of $\Lambda$ by the $G_{n}$. We consider this situation in Section 4.

Theorem 3.1. Let A be a $C^{*}$-algebra, and let

$$
\cdots \stackrel{q_{n+1}}{\longrightarrow} G_{n+1} \stackrel{q_{n}}{\longrightarrow} G_{n} \longrightarrow \cdots \stackrel{q_{1}}{\longrightarrow} G_{1}
$$

be surjective homomorphisms of finite groups. For each $n$ let $\delta^{n}$ be a coaction of $G_{n}$ on A. Suppose that the diagram

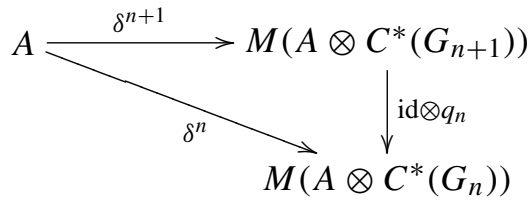

commutes for each $n$.

For each $n$, write $Q_{n}$ for the canonical surjective homomorphism of $\lim \left(G_{m}, q_{m}\right)$ onto $G_{n} ;$ write $q_{n}^{*}: C\left(G_{n}\right) \rightarrow C\left(G_{n+1}\right)$ for the induced map $q_{n}^{*}(f):=\overleftarrow{f} \circ q_{n}$; and write $J_{n}$ for the homomorphism $J_{n}:=j_{A}^{\delta^{n+1}} \times\left(j_{G_{n+1}} \circ q_{n}^{*}\right)$ from $A \times \delta_{\delta^{n}} G_{n}$ to $A \times_{\delta^{n+1}}$ $G_{n+1}$.

Then there is a unique coaction $\delta$ of $\underset{\lim }{\longleftarrow}\left(G_{n}, q_{n}\right)$ on A such that:

(i) the diagrams

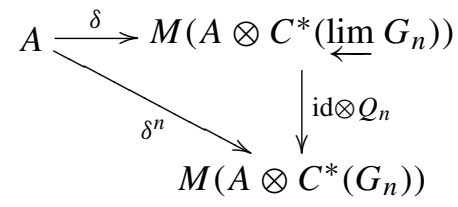

commute; and

(ii) $A \times \delta \lim _{\longleftarrow}\left(G_{n}, q_{n}\right) \cong \underset{\lim }{\longrightarrow}\left(A \times \delta_{\delta^{n}} G_{n}, J_{n}\right)$.

REMARK 3.2. In diagram (3.1) we could replace $M\left(A \otimes C^{*}\left(G_{n}\right)\right)$ with $A \otimes C^{*}\left(G_{n}\right)$ and $M\left(A \otimes C^{*}\left(G_{n+1}\right)\right)$ with $A \otimes C^{*}\left(G_{n+1}\right)$ because $G_{n}, G_{n+1}$ are discrete.

Proof of Theorem 3.1. Put

$$
\begin{aligned}
G & =\lim ^{\leftarrow} G_{n}, \\
B_{n} & =\stackrel{A \times \delta^{n}}{ } G_{n}, \\
J_{n} & =j_{A}^{\delta^{n+1}} \times\left(j_{G_{n+1}} \circ q_{n}^{*}\right): B_{n} \rightarrow B_{n+1}, \\
B & =\underset{\lim }{\longrightarrow}\left(B_{n}, J_{n}\right), \\
K_{n} & =\text { the canonical embedding } B_{n} \rightarrow B .
\end{aligned}
$$

We aim to apply Landstad duality [17]: we will show that $B$ is of the form $C \times{ }_{\delta} G$ for some coaction $(C, G, \delta)$, and then we will show that we can take $C=A$. To apply [17] 
we need:

- $\quad$ an action $\alpha$ of $G$ on $B$; and

- $\quad$ a nondegenerate homomorphism $\mu: C(G) \rightarrow M(B)$ which is $\mathrm{rt}-\alpha$ equivariant, where $\mathrm{rt}$ is the action of $G$ on $C(G)$ by right translation.

Then [17] will provide a coaction $(C, G, \delta)$ and an isomorphism

$$
\theta: B \stackrel{\cong}{\longrightarrow} C \times_{\delta} G
$$

such that

$$
\theta \circ \mu=j_{G} \text { and } \theta\left(B^{\alpha}\right)=j_{C}(C) .
$$

This is simpler than the general construction of [17], because our group $G$ is compact (and then we are really using Landstad's unpublished characterization [13] of crossed products by coactions of compact groups).

We begin by constructing the action $\alpha$ : for each $s \in G$, the diagrams

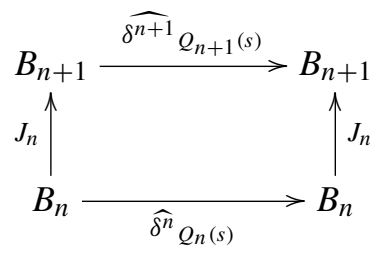

commute because

$$
\begin{aligned}
\widehat{\delta^{n+1}} Q_{n+1}(s) \circ J_{n} \circ j_{A}^{\delta^{n}} & =\widehat{\delta^{n+1}} Q_{n+1}(s) \circ j_{A}^{\delta^{n+1}} \\
& =j_{A}^{\delta^{n+1}} \\
& =J_{n} \circ j_{A}^{\delta^{n}} \\
& =J_{n} \circ \widehat{\delta^{n}} Q_{n}(s) \circ j_{A}^{\delta^{n}}
\end{aligned}
$$

and

$$
\begin{aligned}
\widehat{\delta^{n+1}} Q_{n+1}(s) \circ J_{n} \circ j_{G_{n}} & =\widehat{\delta^{n+1}} Q_{n+1}(s) \circ j_{G_{n+1}} \circ q_{n}^{*} \\
& =j_{G_{n+1}} \circ \mathrm{rt}_{Q_{n+1}(s)} \circ q_{n}^{*} \\
& =j_{G_{n+1}} \circ q_{n}^{*} \circ \mathrm{rt}_{q_{n} \circ Q_{n+1}(s)} \\
& =J_{n} \circ j_{G_{n}} \circ \mathrm{rt}_{Q_{n}(s)} \\
& =J_{n} \circ \widehat{\delta^{n}} Q_{n}(s) \circ j_{G_{n}} .
\end{aligned}
$$

Thus, because the $\widehat{\delta^{n}} Q_{n}(s)$ are automorphisms, by universality there is a unique automorphism $\alpha_{s}$ such that the diagrams

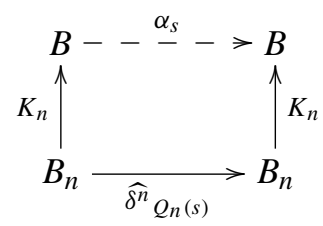


commute. It is easy to check that this gives a homomorphism $\alpha: G \rightarrow$ Aut $B$. We verify continuity: each function $s \mapsto \alpha_{s}(b)$ for $b \in B$ is a uniform limit of functions of the form $s \mapsto \alpha_{s} \circ K_{n}(b)$ for $b \in B_{n}$. But

$$
\alpha_{s} \circ K_{n}(b)=K_{n} \circ{\widehat{\delta^{n}}}_{Q_{n}(s)}(b),
$$

which is continuous since $K_{n}, Q_{n}$, and $t \mapsto \widehat{\delta}^{n}{ }_{t}(b): G_{n} \rightarrow B_{n}$ are.

We turn to the construction of the nondegenerate homomorphism $\mu$ : first note that the increasing union $\bigcup_{n} Q_{n}^{*}\left(C\left(G_{n}\right)\right)$ is dense in $C(G)$ by the Stone-Weierstrass theorem, and it follows that there is an isomorphism

$$
C(G) \cong \underset{\lim }{\longrightarrow}\left(C\left(G_{n}\right), q_{n}^{*}\right),
$$

taking $Q_{n}^{*}$ to the canonical embedding. We have a compatible sequence of nondegenerate homomorphisms

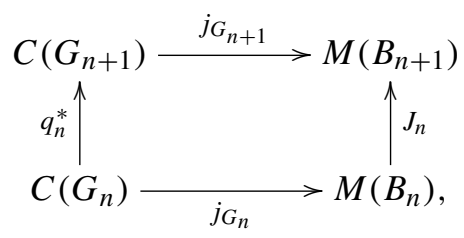

so by universality there is a unique homomorphism $\mu$ making the diagrams

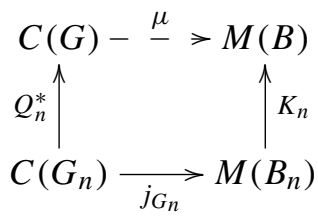

commute. Moreover, $\mu$ is nondegenerate since $K_{n}$ and $j_{G_{n}}$ are.

We now have $\alpha$ and $\mu$, and the equivariance

$$
\alpha_{s} \circ \mu=\mu \circ \mathrm{rt}_{s}
$$

follows from

$$
\begin{aligned}
\alpha_{s} \circ \mu \circ Q_{n}^{*} & =\alpha_{s} \circ K_{n} \circ j_{G_{n}} \\
& =K_{n} \circ \widehat{\delta}^{n} Q_{n}(s) \circ j_{G_{n}} \\
& =K_{n} \circ j_{G_{n}} \circ \mathrm{rt}_{Q_{n}(s)} \\
& =\mu \circ Q_{n}^{*} \circ \mathrm{rt}_{Q_{n}(s)} \\
& =\mu \circ \mathrm{rt}_{s} \circ Q_{n}^{*} .
\end{aligned}
$$

Thus we can apply [17] to obtain a coaction $(C, G, \delta)$ and an isomorphism

$$
\theta: B \stackrel{\cong}{\longrightarrow} C \times \delta G,
$$


such that

$$
\theta \circ \mu=j_{G} \text { and } \theta\left(B^{\alpha}\right)=j_{C}(C) .
$$

We want to take $C=A$. Note that we have a compatible sequence of nondegenerate homomorphisms

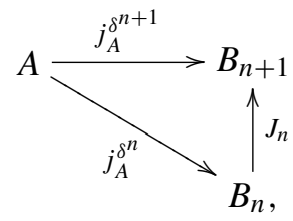

so by universality there is a unique homomorphism $j$ making the diagrams

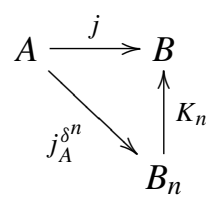

commute. Moreover, $j$ is injective and nondegenerate since $K_{n}$ and $j_{A}^{\delta^{n}}$ are. Because $j, j_{C}$, and $\theta$ are faithful, to show that we can take $C=A$ it suffices to show that

$$
j(A)=B^{\alpha} .
$$

Now

$$
j(A) \subset B^{\alpha}
$$

because

$$
\begin{aligned}
\alpha_{s} \circ j & =\alpha_{s} \circ K_{n} \circ j_{A}^{\delta_{n}} \\
& =K_{n} \circ \widehat{\delta^{n}} Q_{n}(s) \circ j_{A}^{\delta_{n}} \\
& =K_{n} \circ j_{A}^{\delta_{n}} \\
& =j .
\end{aligned}
$$

For the opposite containment, let $b \in B^{\alpha}$. There is a sequence $b_{n} \in B_{n}$ such that $K_{n}\left(b_{n}\right) \rightarrow b$. The functions $s \mapsto \alpha_{s} \circ K_{n}\left(b_{n}\right)$ converge uniformly to the function $s \mapsto \alpha_{s}(b)$, so

$$
\int_{G} \alpha_{s} \circ K_{n}\left(b_{n}\right) d s \rightarrow \int_{G} \alpha_{s}(b) d s=b .
$$

Also

$$
\int_{G} \alpha_{s} \circ K_{n}\left(b_{n}\right) d s=\int_{G} K_{n} \circ \widehat{\delta}^{n} Q_{n}(s)\left(b_{n}\right) d s=K_{n}\left(\int_{G} \widehat{\delta}_{Q_{n}(s)}\left(b_{n}\right) d s\right) .
$$

Since

$$
\int_{G} \widehat{\delta^{n}} Q_{n}(s)\left(b_{n}\right) d s \in B_{n}^{\widehat{\delta^{n}}}=j_{A}^{\delta^{n}}(A)
$$


we conclude that

$$
b \in K_{n} \circ j_{A}^{\delta^{n}}(A)=j(A) .
$$

Therefore we can take $C=A$, so that we have a coaction $(A, G, \delta)$ and an isomorphism

$$
\theta: B \stackrel{\cong}{\longrightarrow} A \times_{\delta} G
$$

such that

$$
\theta \circ \mu=j_{G}
$$

We have proved (ii). For (i), we calculate that

$$
\begin{aligned}
\left(j_{A}^{\delta} \otimes \mathrm{id}\right) \circ\left(\mathrm{id} \otimes Q_{n}\right) \circ \delta & =\left(\mathrm{id} \otimes Q_{n}\right) \circ\left(j_{A}^{\delta} \otimes \mathrm{id}\right) \circ \delta \\
& =\left(\mathrm{id} \otimes Q_{n}\right) \circ \operatorname{Ad}\left(j_{G} \otimes \mathrm{id}\right)\left(w_{G}\right) \circ\left(j_{A}^{\delta} \otimes 1\right) \\
& =\operatorname{Ad}\left(\mathrm{id} \otimes Q_{n}\right)\left(\left(j_{G} \otimes \mathrm{id}\right)\left(w_{G}\right)\right) \circ\left(\mathrm{id} \otimes Q_{n}\right) \circ\left(j_{A}^{\delta} \otimes 1\right) \\
& =\operatorname{Ad}\left(j_{G} \otimes \mathrm{id}\right)\left(\left(\mathrm{id} \otimes Q_{n}\right)\left(w_{G}\right)\right) \circ\left(j_{A}^{\delta} \otimes 1\right) \\
& =\operatorname{Ad}\left(j_{G} \otimes \mathrm{id}\right)\left(\left(Q_{n}^{*} \otimes \mathrm{id}\right)\left(w_{G_{n}}\right)\right) \circ\left(j_{A}^{\delta} \otimes 1\right) \\
& =\operatorname{Ad}\left(j_{G} \circ Q_{n}^{*} \otimes \mathrm{id}\right)\left(w_{G_{n}}\right) \circ\left(j_{A}^{\delta} \otimes 1\right) \\
& =\operatorname{Ad}\left(\theta \circ K_{n} \circ j_{G_{n}} \otimes \mathrm{id}\right)\left(w_{G_{n}}\right) \circ\left(\theta \circ K_{n} \circ j_{A}^{\delta^{n}} \otimes 1\right) \\
& =\left(\theta \circ K_{n} \otimes \mathrm{id}\right) \circ \mathrm{Ad}\left(j_{G_{n}} \otimes \mathrm{id}\right)\left(w_{G_{n}}\right) \circ\left(j_{A}^{\delta^{n}} \otimes 1\right) \\
& =\left(\theta \circ K_{n} \otimes \mathrm{id}\right) \circ\left(j_{A}^{\delta^{n}} \otimes \mathrm{id}\right) \circ \delta^{n} \\
& =\left(\theta \circ K_{n} \circ j_{A}^{\delta^{n}} \otimes \mathrm{id}\right) \circ \delta^{n} \\
& =\left(j_{A}^{\delta} \otimes \mathrm{id}\right) \circ \delta^{n} .
\end{aligned}
$$

Since $j_{A}^{\delta}$ is faithful, we therefore have (id $\left.\otimes Q_{n}\right) \circ \delta=\delta^{n}$.

The following application of Theorem 3.1 motivates the work of the following sections.

EXAmple 3.3. Let $A=C(\mathbb{T})=C^{*}(\mathbb{Z})$, and let $z$ denote the canonical generating unitary function $z \mapsto z$. For $n \in \mathbb{N}$, let $G_{n}:=\mathbb{Z} / 2^{n-1} \mathbb{Z}$ be the cyclic group of order $2^{n-1}$. We write 1 for the canonical generator of $G_{n}$ and 0 for the identity element. Let $g \mapsto u_{n}(g)$ denote the canonical embedding of $G_{n}$ into $C^{*}\left(G_{n}\right)$. Define $q_{n}$ : $G_{n+1} \rightarrow G_{n}$ by $q_{n}(m):=m\left(\bmod 2^{n-1}\right)$, and write $q_{n}$ also for the homomorphism $q_{n}: C^{*}\left(G_{n+1}\right) \rightarrow C^{*}\left(G_{n}\right)$ satisfying $q_{n}\left(u_{n+1}(g)\right)=u_{n}\left(q_{n}(g)\right)$. For each $n$, let $\delta^{n}$ be the coaction of $G_{n}$ on $A$ determined by $\delta^{n}(z):=z \otimes u_{n}(1)$.

Let $g \mapsto u(g)$ denote the canonical embedding of $\lim G_{n}$ as unitaries in the multiplier algebra of $C^{*}\left(\lim ^{\lim } G_{n}\right)$. The coaction $\delta$ of $\varlimsup_{\lim }^{\leftarrow} G_{n}$ on $A$ described in Theorem 3.1 is the one determined by $\delta(z):=z \otimes u(1, \overleftarrow{1, \ldots)}$; the corresponding coaction crossed product is known to be isomorphic to the Bunce-Deddens algebra of type $2^{\infty}$ (see, for example, $[6,8.4 .4]$ ). 


\section{Coverings of skew-products}

In this section and the next, we adopt the following notation and assumptions.

NotATION 4.1. Let $\Lambda$ be a connected row-finite $k$-graph with no sources. Fix a vertex $v \in \Lambda^{0}$, and denote by $\pi \Lambda$ the fundamental group $\pi_{1}(\Lambda, v)$ of $\Lambda$ with respect to $v$. Fix a cocycle $c: \Lambda \rightarrow \pi \Lambda$ such that the skew-product $\Lambda \times_{c} \pi \Lambda$ is isomorphic to the universal covering $\Omega_{\Lambda}$ of $\Lambda$ (such a cocycle exists by [15, Corollary 6.5]).

Fix a descending chain of finite-index normal subgroups

$$
\ldots \triangleleft H_{n+1} \triangleleft H_{n} \triangleleft \ldots \triangleleft H_{1}:=\pi \Lambda \text {. }
$$

For each $n$, let $G_{n}:=\pi \Lambda / H_{n}$, and let $q_{n}: G_{n+1} \rightarrow G_{n}$ be the induced homomorphism

$$
q_{n}\left(g H_{n+1}\right):=g H_{n} .
$$

Then

$$
\cdots \stackrel{q_{n+1}}{\longrightarrow} G_{n+1} \stackrel{q_{n}}{\longrightarrow} G_{n} \longrightarrow \cdots \stackrel{q_{1}}{\longrightarrow} G_{1}:=\{e\}
$$

is a chain of surjective homomorphisms of finite groups. Let $G$ denote the projective $\operatorname{limit}$ group $\lim \left(G_{n}, q_{n}\right)$.

For each $\overleftarrow{n}$, let $c_{n}: \Lambda \rightarrow G_{n}$ be the induced cocycle $c_{n}(\lambda)=c(\lambda) H_{n}$, and let

$$
\Lambda_{n}:=\Lambda \times_{c_{n}} G_{n}
$$

be the skew-product $k$-graph. Define covering maps $p_{n}: \Lambda_{n+1} \rightarrow \Lambda_{n}$ by $p_{n}(\lambda, g):=$ $\left(\lambda, q_{n}(g)\right)$.

As in [15, Theorem 7.1(1)], for each $n$ there is a coaction $\delta^{n}: C^{*}(\Lambda) \rightarrow C^{*}(\Lambda) \otimes$ $C^{*}\left(G_{n}\right)$ determined by $\delta^{n}\left(s_{\lambda}\right):=s_{\lambda} \otimes c_{n}(\lambda)$. Denote by $J_{n}$ the inclusion

$$
J_{n}:=j_{A}^{\delta^{n+1}} \times\left(j_{G_{n+1}} \circ q_{n}^{*}\right): C^{*}(\Lambda) \times_{\delta^{n}} G_{n} \rightarrow C^{*}(\Lambda) \times_{\delta^{n+1}} G_{n+1},
$$

described in Theorem 3.1(ii).

As in [15, Theorem 7.1(ii)], for each $n$ there is an isomorphism $\phi_{n}$ of $C^{*}\left(\Lambda_{n}\right)=$ $C^{*}\left(\Lambda \times_{c_{n}} G_{n}\right)$ onto $C^{*}(\Lambda) \times_{\delta^{n}}\left(G_{n}\right)$ which satisfies $\phi_{n}\left(s_{(\lambda, g)}\right):=\left(s_{\lambda}, g\right)$.

EXAMPLE 4.2 (Example 3.3 continued). Let $\Lambda$ be the path category of the directed graph $B_{1}$ consisting of a single vertex $v$ and a single edge $f$ with $r(f)=s(f)=v$. Note that as a category, $\Lambda$ is isomorphic to $\mathbb{N}$, and the degree functor is then the identity function from $\mathbb{N}$ to itself.

Then $\pi \Lambda$ is the free abelian group generated by the homotopy class of $f$, and so is isomorphic to $\mathbb{Z}$. We define a functor $c: \Lambda \rightarrow \mathbb{Z}$ by $c(f)=1$.

For each $n$, let $H_{n}:=2^{n-1} \mathbb{Z} \subset \mathbb{Z}$, so that $\cdots \triangleleft H_{n+1} \triangleleft H_{n} \triangleleft \cdots \triangleleft H_{1}:=\pi \Lambda$ is a descending chain of finite-index normal subgroups. For each $n, G_{n}:=\mathbb{Z} / H_{n}$ is the cyclic group of order $2^{n-1}$, and $q_{n}: G_{n+1} \rightarrow G_{n}$ is the quotient map described in 
Example 3.3. The induced cocycle $c_{n}: \Lambda \rightarrow G_{n}$ obtained from $c$ is determined by $c_{n}(f)=1 \in \mathbb{Z} / 2^{n-1} \mathbb{Z}$.

For $p \in \mathbb{N}$, let $C_{p}$ denote the simple cycle graph with $p$ vertices: $C_{p}^{0}:=\left\{v_{j}^{p}: j \in\right.$ $\mathbb{Z} / p \mathbb{Z}\}$ and $C_{p}^{1}:=\left\{e_{j}^{p}: j \in \mathbb{Z} / p \mathbb{Z}\right\}$, where $r\left(e_{i}^{p}\right)=v_{i}^{p}$ and $s\left(e_{i}^{p}\right)=v_{i+1}^{p} \bmod p$. For each $n$, the skew-product graph $\Lambda_{n}:=\Lambda \times_{c_{n}} G_{n}$ is isomorphic to the path-category of $C_{2^{n-1}}$. The associated covering map $p_{n}: \Lambda_{n+1} \rightarrow \Lambda_{n}$ corresponds to the doublecovering of $C_{2^{n-1}}$ by $C_{2^{n}}$ satisfying $v_{i}^{2^{n}} \mapsto v_{i}^{2^{n-1} \bmod 2^{n-1}}$ and $e_{i}^{2^{n}} \mapsto e_{i \bmod 2^{n-1}}^{2^{n-1}}$.

Modulo a relabelling of the generators of $\mathbb{N}^{2}$, the 2-graph $\lim \left(\Lambda_{n}, p_{n}\right)$ obtained from this data as in [12] (see Section 2.3) is isomorphic to the 2-graph of [16, Example 6.7]. Combining this with the final observation of Example 3.3, we obtain a new proof that the $C^{*}$-algebra of this 2-graph is Morita equivalent to the BunceDeddens algebra of type $2^{\infty}$ (see [16, Example 6.7] for an alternative proof).

Theorem 4.3. Adopt Notation 4.1. Taking $A:=C^{*}(\Lambda)$, the coactions $\delta^{n}$ and the quotient maps $q_{n}$ make the diagrams (3.1) commute. Let $\delta$ denote the coaction of $G:=\lim _{\leftarrow}\left(G_{n}, q_{n}\right)$ on $C^{*}(\Lambda)$ obtained from Theorem 3.1. Let $P_{0}$ denote the projection $\sum_{v \in \Lambda^{0}} s_{v}$ in the multiplier algebra of $C^{*}\left(\lim \left(\Lambda_{n}, p_{n}\right)\right)$. Then $P_{0}$ is full and

$$
P_{0} C^{*}\left(\stackrel{\lim }{\longleftarrow}\left(\Lambda_{n}, p_{n}\right)\right) P_{0} \cong C^{*}(\Lambda) \times_{\delta} G .
$$

To prove this theorem, we first show that, in the setting described above, the inclusions of $k$-graph algebras induced from the coverings $p_{n}: \Lambda_{n+1} \rightarrow \Lambda_{n}$ as in [12] are compatible with the inclusions of coaction crossed products induced from the quotient maps $q_{n}: G_{n+1} \rightarrow G_{n}$.

Lemma 4.4. With Notation 4.1, fix $n \in \mathbb{N}$, and let $\iota_{p_{n}}$ be the inclusion of $C^{*}\left(\Lambda_{n}\right)$ into $C^{*}\left(\Lambda_{n+1}\right)$ obtained from [12, Proposition 3.3(iv)]. Then the inclusion $\iota_{n}$ and the isomorphisms $\phi_{n}, \phi_{n+1}$ of Notation 4.1 make the following diagram commute:

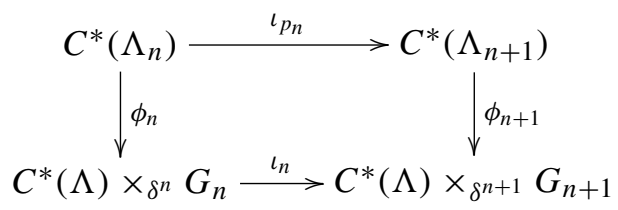

PROOF. By definition,

$$
\iota_{p_{n}}\left(s_{\left(\lambda, g H_{n}\right)}\right)=\sum_{p\left(\lambda^{\prime}, g^{\prime} H_{n+1}\right)=\left(\lambda, g H_{n}\right)} s_{\left(\lambda^{\prime}, g^{\prime} H_{n+1}\right)} .
$$

By definition of $p_{n}$, this becomes

$$
\iota_{p_{n}}\left(s_{\left(\lambda, g H_{n}\right)}\right)=\sum_{\left\{g^{\prime} H_{n+1} \in G_{n+1}: g^{\prime} H_{n}=g H_{n}\right\}} s_{\left(\lambda, g^{\prime} H_{n+1}\right)} .
$$


Hence

$$
\phi_{n+1} \circ \iota_{p_{n}}\left(s_{\left(\lambda, g H_{n}\right)}\right)=\sum_{\left\{g^{\prime} H_{n+1} \in G_{n+1}: g^{\prime} H_{n}=g H_{n}\right\}}\left(s_{\lambda}, g^{\prime} H_{n+1}\right) .
$$

But this is precisely $\iota\left(\phi_{n}\left(s_{\left(\lambda, g H_{n}\right)}\right)\right)$ by definition of $\iota$ and $\phi_{n}$.

Corollary 4.5. With Notation 4.1, let $P_{0}$ denote the projection $\sum_{v \in \Lambda^{0}} s_{v}$ in the multiplier algebra of $C^{*}\left(\lim \left(\Lambda_{n}, p_{n}\right)\right)$. Then $P_{0}$ is full and

$$
P_{0} C^{*}\left(\stackrel{\lim }{\longleftarrow}\left(\Lambda_{n}, p_{n}\right)\right) P_{0} \cong \underset{\lim }{\longrightarrow}\left(C^{*}(\Lambda) \times_{\delta^{n}} G_{n}, \iota_{n}\right) .
$$

Proof. By [12, Equation (3.2)], $P_{0} C^{*}\left(\lim \left(\Lambda_{n}, p_{n}\right)\right) P_{0}$ is isomorphic to $\stackrel{\lim }{\longrightarrow}\left(C^{*}\left(\Lambda_{n}\right), \iota_{p_{n}}\right)$. The latter is isomorphic to $\left.\underset{\lim }{\longrightarrow} C^{*}(\Lambda) \times \delta^{n} G_{n}, \iota_{n}\right)$ by Lemma 4.4 $\overrightarrow{\text { and }}$ the universal property of the direct limit.

Proof of THEOREM 4.3. It is immediate from the definitions of the maps involved that the maps $\delta^{n}$ and $q_{n}$ make the diagram (3.1) commute. The rest of the statement then follows from Corollary 4.5 and Theorem 3.1(ii).

\section{Simplicity}

In this section we frequently embed $\mathbb{N}^{k}$ into $\mathbb{N}^{k+1}$ as the subset consisting of elements whose $(k+1)$ th coordinate is equal to zero. For $n \in \mathbb{N}^{k}$, we write $(n, 0)$ for the corresponding element of $\mathbb{N}^{k+1}$.

TheOrem 5.1. Adopt Notation 4.1. The $(k+1)$-graph $C^{*}$-algebra

$C^{*}\left(\lim \left(\Lambda_{n}, p_{n}\right)\right)$ is simple if and only if the following two conditions are satisfied:

(i) each $\Lambda_{n}$ is cofinal;

(ii) whenever $v \in \Lambda^{0}, p \neq q \in \mathbb{N}^{k}$ satisfy $\sigma^{p}(x)=\sigma^{q}(x)$ for all $x \in v \Lambda^{0}$, there exist $x \in v \Lambda^{\infty}, l \in \mathbb{N}^{k}$ and $N \in \mathbb{N}$ such that $c_{N}(x(p, p+l)) \neq c_{N}(x(q, q+l))$.

The idea is to prove the theorem by appealing to [18, Theorem 3.1]. To do this, we will first describe the infinite paths in $\lim \left(\Lambda_{n}, p_{n}\right)$. We identify $\underset{\lim }{\leftarrow}\left(G_{n}, q_{n}\right)$ with the set of sequences $g=\left(g_{n}\right)_{n=1}^{\infty}$ such that $\bar{q}_{n}\left(g_{n+1}\right)=g_{n}$ for all $n$.

Lemma 5.2. Adopt Notation 4.1. Fix $x \in \Lambda^{\infty}$ and $g=\left(g_{n}\right)_{n=1}^{\infty} \in \lim ^{\infty}\left(G_{n}, q_{n}\right)$. For each $n \in \mathbb{N}$ there is a unique infinite path $\left(x, g_{n}\right) \in \Lambda_{n}^{\infty}$ determined by $\left(x, g_{n}\right)(0, m)=\left(x(0, m), c_{n}(x(0, m))^{-1} g_{n}\right)$ for all $m \in \mathbb{N}^{k}$. There is a unique infinite path $x^{g} \in\left(\lim \left(\Lambda_{n}, p_{n}\right)\right)^{\infty}$ such that $x^{g}(0,(m, 0))=x(0, m)$ for all $m \in$ $\mathbb{N}^{k}$ and $x^{g}\left(n e_{k+1}\right)=\left(x(0), g_{n}\right)$ for all $n \in \mathbb{N} ;$ moreover, $\sigma^{n e_{k+1}}\left(x^{g}\right)(0,(m, 0))=$ $\left(x, g_{n}\right)(0, m)$ for all $m \in \mathbb{N}^{k}$. Finally, every infinite path $y \in\left(\lim \left(\Lambda_{n}, p_{n}\right)\right)^{\infty}$ is of the form $\sigma^{n e_{k+1}}\left(x^{g}\right)$ for some $n \in \mathbb{N}, x \in \Lambda^{\infty}$ and $g \in \underset{\lim }{\longleftarrow}\left(G_{n}, q_{n}\right)$. 
Proof. That the formula given determines unique infinite paths $\left(x, g_{n}\right), n \in \mathbb{N}$, follows from [9, Remarks 2.2]. That there is a unique infinite path $x^{g}$ such that $x^{g}(0,(m, 0))=x(0, m)$ for all $m \in \mathbb{N}^{k}$ and $x^{g}\left(n e_{k+1}\right)=\left(x(0), g_{n}\right)$ for all $n \in \mathbb{N}$ follows from the observation that for each $n \in \mathbb{N}$ there is a unique path

$$
\alpha=\alpha_{g, n}:=e\left(x(0), g_{1}\right) e\left(x(0), g_{2}\right) \cdots e\left(x(0), g_{n}\right),
$$

with $d\left(\alpha_{g, n}\right)=n e_{k+1}, r(\alpha)=x(0) \in \Lambda^{0}$ and $s(\alpha)=\left(x(0), g_{n}\right) \in \Lambda_{n}^{0}$, and that for each $m \in \mathbb{N}^{k}$,

$$
\begin{array}{r}
\alpha\left(x, g_{n}\right)(0, m)=x(0, m) e\left(x(m), c_{1}(x(0, m))^{-1} g_{1}\right) \\
\cdots e\left(x(m), c_{n}(x(0, m))^{-1} g_{n}\right)
\end{array}
$$

is the unique minimal common extension of $x(0, m)$ and $\alpha$. This also establishes the assertion that $\sigma^{n e_{k+1}}\left(x^{g}\right)(0,(m, 0))=\left(x, g_{n}\right)(0, m)$ for all $m \in \mathbb{N}^{k}$.

For the final assertion, fix $y \in\left(\lim \left(\Lambda_{n}, p_{n}\right)\right)^{\infty}$. We must have $y(0)=\left(v, g_{n}\right)$ for some $v \in \Lambda^{0}, g_{n} \in G_{n}=\pi \Lambda / \widetilde{H_{n}}$ and $n \in \mathbb{N}$. Let $x \in \Lambda_{n}^{\infty}$ be the infinite path determined by $x(0, m):=y(0,(m, 0))$ for all $m \in \mathbb{N}^{k}$. By definition of $\Lambda_{n}=\Lambda \times_{c_{n}}$ $G_{n}$, we have $x(0, m):=\left(\alpha_{m}, c_{n}\left(\alpha_{m}\right)^{-1} g_{n}\right)$ where each $\alpha_{m} \in v \Lambda^{m}$ and $g$ is the element of $\pi \Lambda$ such that $y(0)=v\left(g_{n}\right)$ as above. There is then an infinite path in $x^{\prime} \in \Lambda^{\infty}$ determined by $x^{\prime}(0, m)=\alpha_{m}$ for all $m \in \mathbb{N}^{k}$. For $n>i \geq 1$, inductively define $g_{i}:=q_{i}\left(g_{i+1}\right)$, and for $n<i$ let $g_{i}$ be the unique element of $G_{i}$ such that $y\left((i-n) e_{k+1}\right)=\left(v, g_{i}\right)$; that such $g_{i}$ exist follows from the definition of $\lim \left(\Lambda_{n}, p_{n}\right)$. Then $g:=\left(g_{i}\right)_{i=1}^{\infty}$ is an element of $\lim \left(G_{n}, q_{n}\right)$ by definition, and routine calculations using the definitions of the $\Lambda_{n}$ show that $x=\sigma^{n e_{k+1}}\left(\left(x^{\prime}\right)^{g}\right)$.

Lemma 5.3. Adopt Notation 4.1. Then the $(k+1)$-graph $\lim \left(\Lambda_{n}, p_{n}\right)$ is cofinal if and only if each $\Lambda_{n}$ is cofinal.

Proof. Suppose that each $\Lambda_{n}$ is cofinal. Fix $y \in \lim \left(\Lambda_{n}, p_{n}\right)$ and $w \in \lim \left(\Lambda^{0}\right)$. By Lemma 5.2, we have $y=\sigma^{i_{0} e_{k+1}}\left(x^{g}\right)$ for some $g=\left(g_{n}\right)_{n=1}^{\infty} \in \underset{\lim }{\leftarrow}\left(\overleftarrow{G_{n}}, q_{n}\right)$, some $i_{0} \in \mathbb{N}$ and some $x \in \Lambda^{\infty}$. We must show that $w\left(\lim \left(\Lambda_{n}, p_{n}\right)\right) \overleftarrow{y(q)} \neq \emptyset$ for some $q$. We have $w \in \Lambda_{m}^{0}$ for some $m \in \mathbb{N}$, so $w=\left(w^{\prime}, \leftarrow_{h}\right)$ for some $h \in G_{m}$. If $m<i_{0}$, fix any $h^{\prime} \in \pi \Lambda$ such that $h^{\prime} H_{i_{0}}=h$, and note that $w\left(\lim \left(\Lambda_{n}, p_{n}\right)\right)\left(w^{\prime}, h H_{i_{0}}\right)$ is nonempty, so that it suffices to show that $\left.\left(w^{\prime}, h^{\prime} H_{i_{0}}\right)\left(\lim {\overleftarrow{(}\left(\Lambda_{n}\right.}_{n}, p_{n}\right)\right) y(q) \neq \emptyset$ for some $q$. That is to say, we may assume without loss of generality that $m \geq i_{0}$. But now $w \in \Lambda_{m}^{0}$ and $\sigma^{\left(0, \ldots, 0, m-i_{0}\right)}(y) \in\left(\lim \left(\Lambda_{n}, p_{n}\right)\right)^{\infty}$ with $r(y) \in \Lambda_{i_{0}}^{0}$. Since $\Lambda_{n}$ is cofinal, we have $w \Lambda_{i_{0}}\left(x, g_{m}\right)(q) \neq \emptyset$ for some $q \in \mathbb{N}^{k}$ (recall that $x,\left(g_{i}\right)_{i=1}^{\infty}$ are such that $\left.y=\sigma^{i_{0} e_{k+1}}\left(x^{g}\right)\right)$. By definition, $\left(x, g_{m}\right)(q)=y\left(q_{1}, \ldots, q_{k}, m-i_{0}\right)$ and this shows that $w\left(\lim \left(\Lambda_{n}, p_{n}\right)\right) y(q) \neq \emptyset$ for $q=\left(q_{1}, \ldots, q_{k}, m-n\right)$.

Now suppose that $\lim \left(\Lambda_{n}, p_{n}\right)$ is cofinal. Fix $n \in \mathbb{N}$ and a vertex $w$ and an infinite path $x$ in $\Lambda_{n}$. Then $x(0)=\left(v, g H_{n}\right)$ for some $v \in \Lambda^{0}, g \in \pi \Lambda$. There are 
paths $\alpha_{m} \in \Lambda_{n}^{m}, m \in \mathbb{N}^{k}$, determined by $x(0, m)=\left(\alpha_{m}, c_{n}\left(\alpha_{m}\right)^{-1} g H_{n}\right)$; there is then an infinite path $x^{\prime} \in \Lambda^{\infty}$ such that $x^{\prime}(0, m)=\alpha_{m}$ for all $m$. Let $g_{i}:=g H_{i}$ for all $i \in \mathbb{N}$. In an abuse of notation we denote by $g$ the element $\left(g H_{i}\right)_{i=1}^{\infty}$ of $\lim \left(G_{n}, q_{n}\right)$. Let $y=\sigma^{n}\left(\left(x^{\prime}\right)^{g}\right)$ be the infinite path of $\lim \left(\Lambda_{n}, p_{n}\right)$ provided by

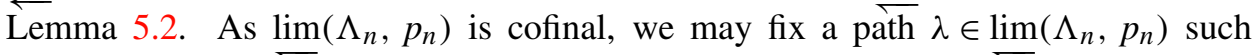

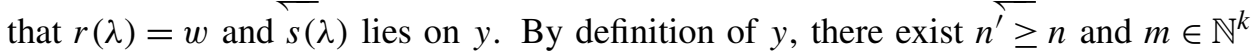
such that $s(\lambda)=\left(x^{\prime}(m), c_{n^{\prime}}\left(\alpha_{m}\right)^{-1} g_{n^{\prime}}\right)$. We then have $d(\lambda)_{k+1}=n^{\prime}-n$, and we may factorize $\lambda=\lambda^{\prime} \lambda^{\prime \prime}$ where $d\left(\lambda^{\prime}\right)=d(\lambda)-\left(n^{\prime}-n\right) e_{k+1}$ and $d\left(\lambda^{\prime \prime}\right)=\left(n^{\prime}-n\right) e_{k+1}$. By construction of $\lim \left(\Lambda_{n}, p_{n}\right)$, if $d(\mu)=j e_{k+1}$ and $s(\mu)=\left(v, g H_{n}\right) \in \Lambda_{n}^{0}$ then $n \geq j$ and $r(\mu)=\left(v, \overleftarrow{g H}_{n-j}\right) \in \Lambda_{n-j}^{0}$. In particular,

$$
s\left(\lambda^{\prime}\right)=r\left(\lambda^{\prime \prime}\right)=\left(x^{\prime}(m), c_{n}\left(\alpha_{m}\right)^{-1} g_{n}\right)=x(m),
$$

so $w \Lambda_{n} x(m) \neq \emptyset$.

Lemma 5.4. Adopt Notation 4.1. Then the $(k+1)$-graph $\lim \left(\Lambda_{n}, p_{n}\right)$ has no local periodicity if and only if it satisfies condition (ii) of Theorem $\overline{5.1}$.

PROOF. First suppose that condition (ii) of Theorem 5.1 holds. Fix a vertex $v \in$ $\left(\lim \left(\Lambda_{n}, p_{n}\right)\right)^{0}$ and $p \neq q \in \mathbb{N}^{k+1}$. So $v \in \Lambda_{n}^{0}$ for some $n$, and $v$ therefore has the form $v=\left(w, g H_{n}\right)$ for some $w \in \Lambda^{0}$ and $g \in \pi \Lambda$. We must show that there exists $x \in v\left(\lim \left(\Lambda_{n}, p_{n}\right)\right)^{\infty}$ such that $\sigma^{p}(x) \neq \sigma^{q}(x)$.

We first consider the case where $p_{k+1} \neq q_{k+1}$. By construction of the tower graph $\lim \left(\Lambda_{n}, p_{n}\right)$, this forces the vertices $x(p)$ and $x(q)$ to lie in distinct $\Lambda_{n}$ for any

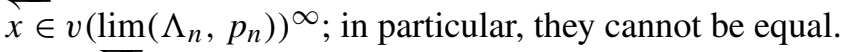

Now suppose that $p_{k+1}=q_{k+1}$. If every $x \in v\left(\lim \left(\Lambda_{n}, p_{n}\right)\right)^{\infty}$ satisfies $\sigma^{p}(x)=$ $\sigma^{q}(x)$, then for any $\alpha \in v\left(\lim \left(\Lambda_{n}, p_{n}\right)\right)^{p_{k+1} e_{k+1}}$ and any $y \in s(\alpha)\left(\lim \left(\Lambda_{n}, p_{n}\right)\right)^{\infty}$, we have $\sigma^{p}(\alpha y)=\sigma^{q}(\alpha y)$; that is,

$$
\sigma^{p-p_{k+1} e_{k+1}}(y)=\sigma^{q-q_{k+1} e_{k+1}}(y) \quad \text { for all } y \in s(\alpha)\left(\underline{\lim }\left(\Lambda_{n}, p_{n}\right)\right)^{\infty} .
$$

So we may assume without loss of generality that $p_{k+1}=q_{k+1}=0$. Write $p^{\prime}$ and $q^{\prime}$ for the elements of $\mathbb{N}^{k}$ whose entries are the first $k$ entries of $p$ and $q$.

We have $v \in \Lambda_{n}$ for some $n$, so there exist $w \in \Lambda^{0}$ and $g \in \pi \Lambda$ such that $v=\left(w, g H_{n}\right)$. Suppose first that there exists $x \in w \Lambda^{\infty}$ such that $\sigma^{p^{\prime}}(x) \neq \sigma^{q^{\prime}}(x)$. Then the infinite path $\left(x, g H_{n}\right) \in v \Lambda_{n}^{\infty}$ such that

$$
\left(x, g H_{n}\right)(0, m):=\left(x(0, m), c_{n}(x(0, m))^{-1} g H_{n}\right) \quad \text { for all } m \in \mathbb{N}^{k},
$$

also satisfies $\sigma^{p^{\prime}}\left(\left(x, g H_{n}\right)\right) \neq \sigma^{q^{\prime}}\left(\left(x, g H_{n}\right)\right)$. By Lemma 5.2 we may choose an infinite path $y$ such that $\left.y\right|_{\mathbb{N}^{k} \times\{0\}}=\left(x, g H_{n}\right)$, and then $y \in v\left(\lim \left(\Lambda_{n}, p_{n}\right)\right)^{\infty}$ satisfies $\sigma^{p}(y) \neq \sigma^{q}(y)$. 
Now suppose that every path $x \in w \Lambda^{\infty}$ satisfies $\sigma^{p^{\prime}}(x)=\sigma^{q^{\prime}}(x)$. Then by condition (ii) of Theorem 5.1, we may fix $x \in w \Lambda^{\infty}$ and $N \in \mathbb{N}$ such that $c_{N}\left(x\left(0, p^{\prime}\right)\right) \neq c_{N}\left(x\left(0, q^{\prime}\right)\right)$. It then follows from the definition of the $c_{j}$ that $c_{j}\left(x\left(0, p^{\prime}\right)\right) \neq c_{j}\left(x\left(0, q^{\prime}\right)\right)$ whenever $j \geq N$. So with $j:=\max \{N, n\}$,

$$
\begin{aligned}
\left(x, g H_{j}\right)\left(p^{\prime}\right) & =\left(x\left(p^{\prime}\right), c_{j}\left(x\left(0, p^{\prime}\right)\right)^{-1} g H_{j}\right) \neq\left(x\left(q^{\prime}\right), c_{j}\left(x\left(0, q^{\prime}\right)\right)^{-1} g H_{j}\right) \\
& =\left(x, g H_{j}\right)\left(q^{\prime}\right) .
\end{aligned}
$$

There is an element $g=\left(g_{i}\right)_{i=1}^{\infty}$ of $\lim \left(G_{n}, q_{n}\right)$ determined by $g_{i}:=g H_{i}$ for all $i$. Let

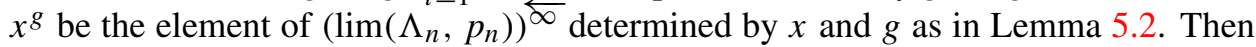
$\left(x, g H_{n}\right)\left((j-n) e_{k+1}+p\right) \neq\left(x, g H_{n}\right)\left((j-n) e_{k+1}+q\right)$, and therefore $x^{g}$ satisfies $\sigma^{p}\left(x^{g}\right) \neq \sigma^{q}\left(x^{g}\right)$ as required. Hence condition (ii) of Theorem 5.1 implies that $\lim \left(\Lambda_{n}, p_{n}\right)$ has no local periodicity.

To show that if $\lim \left(\Lambda_{n}, p_{n}\right)$ has no local periodicity then condition (ii) of Theorem 5.1 holds, we prove the contrapositive statement. Suppose that condition (ii) of Theorem 5.1 does not hold. Fix $v \in \Lambda^{0}$ and $p, q \in \mathbb{N}^{k}$ such that $\sigma^{p}(x)=\sigma^{q}(x)$ for all $x \in v \Lambda^{\infty}$ and $c_{n}(x(p, p+l))=c_{n}(x(q, q+l))$ for all $n \in \mathbb{N}, l \in \mathbb{N}^{k}$. Then for each $x \in v \Lambda^{\infty}$ and each $g=\left(g_{n}\right)_{n=1}^{\infty} \in \lim \left(G_{n}, p_{n}\right)$, we have $\sigma^{p}\left(x, g_{n}\right)(0, l)=$ $\sigma^{q}\left(x, g_{n}\right)(0, l)$ for all $n \in \mathbb{N}$ and $l \in \mathbb{N}^{k}$. Hence Lemma 5.2 implies that every $y \in v\left(\lim \left(\Lambda_{n}, p_{n}\right)\right)^{\infty}$ satisfies $\sigma^{(p, 0)}(y)=\sigma^{(q, 0)}(y)$.

Proof of Theorem 5.1. From [18, Theorem 3.1] we see that $C^{*}\left(\lim \left(\Lambda_{n}, p_{n}\right)\right)$ is simple if and only if $\lim \left(\Lambda_{n}, p_{n}\right)$ is cofinal and has no local periodicity. The result then follows directly from Lemmas 5.3 and 5.4.

\section{Projective limit $k$-graphs}

Let $\left(\Lambda_{n}, \Lambda_{n+1}, p_{n}\right)_{n=1}^{\infty}$ be a sequence of row-finite coverings of $k$-graphs with no sources as in Section 2.3. We aim to show that the sets $\left(\underset{\lim }{\longleftarrow} \Lambda_{i}\right)^{m}:=\lim \left(\Lambda_{i}^{m}, p_{i}\right)$ under the projective limit topology with the natural (coordinate-wise) range and source maps specify a topological $k$-graph (in the sense of Yeend). Moreover, we show that the associated topological $k$-graph $C^{*}$-algebra is isomorphic to the full corner $P_{0} C^{*}\left(\lim \left(\Lambda_{n} ; p_{n}\right)\right) P_{0}$ determined by $P_{0}:=\sum_{v \in \Lambda_{1}^{0}} s_{v}$. In particular, when the $\Lambda_{n}$ and $p_{n}$ are as in Notation 4.1 , the $C^{*}$-algebra of the projective limit topological $k$ graph is isomorphic to the crossed product of $C^{*}(\Lambda)$ by the coaction of the projective limit of the groups $G_{i}$ obtained from Theorem 3.1.

Let $\left(\Lambda_{n}, \Lambda_{n+1}, p_{n}\right)_{n=1}^{\infty}$ be a sequence of row-finite coverings of $k$-graphs with no sources. Let $\lim _{(}\left(\Lambda_{i}, p_{i}\right)$ be the projective limit category, equipped with the projective limit topology. That is, $\underset{\leftarrow}{\lim }\left(\Lambda_{i}, p_{i}\right)$ consists of all sequences $\left(\lambda_{i}\right)_{i=1}^{\infty}$ such that each $\lambda_{i} \in \Lambda_{i}$ and $p_{i}\left(\lambda_{i+1}\right)=\overleftarrow{\lambda_{i}}$; the structure maps $\tilde{r}, \tilde{s}$, $\tilde{o}$ and $\tilde{\text { id }}$ on $\lim \left(\Lambda_{i}, p_{i}\right)$ are obtained by pointwise application of the corresponding structure maps for $\Lambda$. The cylinder sets $Z\left(\lambda_{1}, \ldots, \lambda_{j}\right):=\left\{\left(\mu_{i}\right)_{i=1}^{\infty} \in \lim \left(\Lambda_{i}, p_{i}\right): \mu_{i}=\lambda_{i}\right.$ for $\left.1 \leq i \leq j\right\}$ form a basis of compact open sets for a locally compact Hausdorff topology. 
Define $\tilde{d}: \lim _{(}\left(\Lambda_{i}, p_{i}\right) \rightarrow \mathbb{N}^{k}$ by $\tilde{d}\left(\left(\lambda_{i}\right)_{i=1}^{\infty}\right):=d\left(\lambda_{1}\right)$. Since the $p_{i}$ are degreepreserving,

$$
\tilde{d}\left(\left(\lambda_{i}\right)_{i=1}^{\infty}\right)=d\left(\lambda_{i}\right) \quad \text { for all } i \geq 1 .
$$

For fixed $\lambda=\left(\lambda_{i}\right)_{i=1}^{\infty} \in \lim _{\leftarrow}\left(\Lambda_{i}, p_{i}\right)^{m+n}$, the unique factorization property for each $\lambda_{i}$ produces unique elements $\lambda(0, m):=\left(\lambda_{i}(0, m)\right)_{i=1}^{\infty} \in \underset{\lim }{\longleftarrow}\left(\Lambda_{i}, p_{i}\right)^{m}$ and $\lambda(m, n):=$ $\left(\lambda_{i}(m, n)\right)_{i=1}^{\infty} \in \lim \left(\Lambda_{i}, p_{i}\right)^{n}$ such that $\lambda=\lambda(0, m) \lambda(m, n)$; that is, $\left(\lim \left(\Lambda_{i}, p_{i}\right), \tilde{d}\right)$ is a second-countable small category with a degree functor satisfying the factorization property.

The identity $\tilde{d}\left(\left(\lambda_{i}\right)_{i=1}^{\infty}\right)=d\left(\lambda_{i}\right)$ for all $i \geq 1$ implies that $Z\left(\lambda_{1}, \ldots, \lambda_{j}\right)$ is empty unless $d\left(\lambda_{1}\right)=\cdots=d\left(\lambda_{j}\right)$, and it follows that $\tilde{d}$ is continuous.

We claim that $\tilde{r}$ and $\tilde{s}$ are local homeomorphisms. To see this, fix a cylinder set $Z\left(v_{1}, \ldots, v_{j}\right) \subset \lim _{\leftarrow}\left(\Lambda_{i}, p_{i}\right)^{0}$ and, for $\lambda \in v_{1} \Lambda_{1}$ and $2 \leq l \leq j$, let $v_{l} p_{1, l}^{-1}(\lambda)$ be the unique element of $v_{l} \Lambda_{l}$ such that $p_{1} \circ p_{2} \circ \cdots \circ p_{l-1}\left(v_{l} p_{1, l}^{-1}(\lambda)\right)=\lambda$. Then

$$
\tilde{r}^{-1}\left(Z\left(v_{1}, \ldots, v_{j}\right)\right) \cap \lim _{\longleftarrow}\left(\Lambda_{i}, p_{i}\right)^{n}:=\bigsqcup_{\lambda \in v_{1} \Lambda_{1}^{n}} Z\left(\lambda, v_{2} p_{1,2}^{-1}(\lambda), \ldots, v_{j} p_{1, j}^{-1}(\lambda)\right),
$$

which is clearly open, showing that $\tilde{r}$ is continuous. Moreover, this same formula shows that for $\lambda=\left(\lambda_{i}\right)_{i=1}^{\infty} \in \lim _{(}\left(\Lambda_{i}, p_{i}\right)$, the restriction of $\tilde{r}$ to $Z\left(\lambda_{1}\right)$ is a homeomorphism, and $\tilde{r}$ is a local homeomorphism as claimed. A similar argument shows that $\tilde{s}$ is also a local homeomorphism.

It is easy to see that the inverse image under composition of the cylinder set $Z\left(\lambda_{1}, \ldots, \lambda_{j}\right) \in \lim \left(\Lambda_{i}, p_{i}\right)^{n}$ is equal to the disjoint union

$$
\bigsqcup_{p+q=n} Z\left(\lambda_{1}(0, p), \ldots, \lambda_{j}(0, p)\right) \times Z\left(\lambda_{1}(p, q), \ldots, \lambda_{j}(p, q)\right),
$$

of cartesian products of cylinder sets and hence is open, so that composition is continuous, and it follows that $\left(\lim \left(\Lambda_{i}, p_{i}\right), \tilde{d}\right)$ is a topological $k$-graph in the sense of Yeend $[20,21]$.

Let $\lim \left(\Lambda_{n} ; p_{n}\right)$ be as described in Section 2.3, and let $P_{0}$ denote the full projection $\sum_{v \in \Lambda_{1}^{0}} \overleftarrow{s_{v}} \in M\left(C^{*}\left(\lim \left(\Lambda_{n} ; p_{n}\right)\right)\right)$. For the following proposition, we need to describe $P_{0} C^{*}\left(\lim \left(\Lambda_{n} ; p_{n}\right)\right) \overbrace{P_{0}}$ in detail. For $n \geq m \geq 1$, we write $p_{m, n}: \Lambda_{n} \rightarrow \Lambda_{m}$ for the covering map $p_{m, n}:=p_{m} \circ \cdots \circ p_{n-1}$, with the convention that $p_{n, n}$ is the identity map on $\Lambda_{n}$. For $v \in \Lambda_{m}^{0}$, and $l \leq m$, we denote by $\alpha_{l, m}(v)$ the unique path in $\lim \left(\Lambda_{n} ; p_{n}\right)^{(m-l) e_{k+1}}$ whose source is $v$ (and whose range is $p_{l, m}(v)$ ). In particular, $\overleftarrow{\alpha}_{1, m}(v)$ the unique path in $\lim \left(\Lambda_{n} ; p_{n}\right)^{(m-1) e_{k+1}}$ whose source is $v$ with range in $\Lambda_{1}$. For $\lambda \in \Lambda_{m}$,

$$
\begin{aligned}
s_{\alpha_{1, m}(r(\lambda))} s_{\alpha_{1, m}(r(\lambda))}^{*} s_{p_{1, m}(\lambda)} & =s_{\alpha_{1, m}(r(\lambda))} s_{\lambda} s_{\alpha_{1, m}(s(\lambda))}^{*} \\
& =s_{p_{1, m}(\lambda)} s_{\alpha_{1, m}(s(\lambda))} s_{\alpha_{1, m}(s(\lambda))}^{*}
\end{aligned}
$$


Furthermore, $P_{0} C^{*}\left(\lim \left(\Lambda_{n}, p_{n}\right)\right) P_{0}$ is equal to the closed span

$$
P_{0} C^{*}\left(\underset{\lim }{\longleftarrow}\left(\Lambda_{n}, p_{n}\right)\right) P_{0}=\overline{\operatorname{span}}\left\{s_{\alpha_{1, m}(r(\lambda))} s_{\lambda} s_{\alpha_{1, m}(s(\lambda))}^{*}: m \geq 1, \lambda \in \Lambda_{m}\right\} .
$$

PROPOSITION 6.1. Let $\left(\Lambda_{n}, \Lambda_{n+1}, p_{n}\right)_{n=1}^{\infty}$ be a sequence of row-finite coverings of $k$-graphs with no sources, and let $\lim \left(\Lambda_{n} ; p_{n}\right)$ be the associated $(k+1)$-graph as in [12]. Let $P_{0}:=\sum_{v \in \Lambda_{1}^{0}} s_{v} \in M C^{*}\left(\lim \left(\Lambda_{n} ; p_{n}\right)\right)$. Let $\left(\lim _{\leftarrow}\left(\Lambda_{i}, p_{i}\right), \tilde{d}\right)$ be the topological $k$-graph defined above. Then there is a unique isomorphism

$$
\pi: P_{0} C^{*}\left(\stackrel{\lim }{\longleftarrow}\left(\Lambda_{n}, p_{n}\right)\right) P_{0} \rightarrow C^{*}\left(\lim _{\longleftarrow}\left(\Lambda_{i}, p_{i}\right)\right),
$$

such that for $\lambda \in \Lambda_{m}$,

$$
\pi\left(s_{\alpha_{1, m}(r(\lambda))} s_{\lambda} s_{\alpha_{1, m}(s(\lambda))}^{*}\right)=\chi Z\left(p_{1, m}(\lambda), p_{2, m}(\lambda), \ldots, p_{m-1, m}(\lambda), \lambda\right) .
$$

In particular, with Notation 4.1, there is an isomorphism of the $C^{*}$-algebra $C^{*}\left(\lim _{\longleftarrow}\left(\Lambda_{i}, p_{i}\right)\right)$ of the topological $k$-graph $\lim _{\longleftarrow}\left(\Lambda_{i}, p_{i}\right)$ with the coaction crossed product $C^{*}(\Lambda) \times{ }_{\delta} G$.

PROOF. The final statement will follow from Theorem 4.3 once we establish the first statement.

To prove the first statement we will use Allen's gauge-invariant uniqueness theorem for corners in $k$-graph algebras [1]. We adopt Allen's notation: for $\mu, v \in$ $\Lambda_{1}^{0} \lim \left(\Lambda_{n} ; p_{n}\right)$, we let $t_{\mu, v}:=s_{\mu} s_{v}^{*} \in P_{0} C^{*}\left(\underline{\lim }\left(\Lambda_{n} ; p_{n}\right)\right) P_{0}$. The factorization property guarantees that for $\mu, v \in \Lambda_{1}^{0} \lim \left(\Lambda_{n} ; \widehat{p_{n}}\right)$, we can rewrite $\mu=\alpha_{1, m}\left(r\left(\mu^{\prime}\right)\right) \mu^{\prime}$

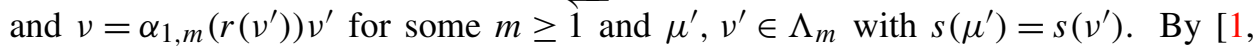
Corollary 3.7], there is an isomorphism $\theta$ of $P_{0} C^{*}\left(\lim \left(\Lambda_{n} ; p_{n}\right)\right) P_{0}$ onto Allen's universal algebra $C^{*}\left(\lim \left(\Lambda_{n} ; p_{n}\right), \Lambda_{1}^{0}\right)$ (see [1, Definition 3.1 and the following paragraphs]) which satisfies $\theta\left(t_{\mu, v}\right)=T_{\mu, \nu}$ for all $\mu, v$. It therefore suffices to show that there is an isomorphism $\psi: C^{*}\left(\lim \left(\Lambda_{n} ; p_{n}\right), \Lambda_{1}^{0}\right) \rightarrow C^{*}\left(\lim _{\longleftarrow}\left(\Lambda_{i}, p_{i}\right)\right)$

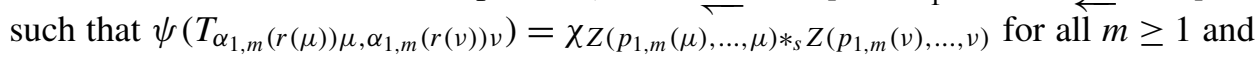
$\mu, \nu \in \Lambda_{m}$ with $s(\mu)=s(\nu)$; the composition $\pi:=\psi \circ \theta$ clearly satisfies (6.1), and it is uniquely specified by (6.1) because the elements $\left\{t_{\alpha_{1, m}(r(\lambda)) \lambda, \alpha_{1, m}(s(\lambda))}\right.$ : $\left.m \geq 1, \lambda \in \Lambda_{m}\right\}$ generate $P_{0} C^{*}\left(\lim \left(\Lambda_{n} ; p_{n}\right)\right) P_{0}$ as a $C^{*}$-algebra.

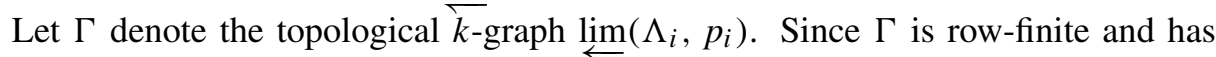
no sources, $\partial \Gamma=\Gamma^{\infty}$. As in [21], for open subsets $U, V \subset \Gamma$, let $Z_{\mathcal{G}_{\Gamma}}\left(U *_{s} V, m\right)$ denote the set $\left\{(\mu x, m, v x): \mu \in U, v \in V, x \in \Gamma^{\infty}, s(\mu)=s(v)=r(x)\right\}$. Then $\mathcal{G}_{\Gamma}$ is the locally compact Hausdorff topological groupoid

$$
\mathcal{G}_{\Gamma}=\left\{(x, m-n, y): x, y \in \Gamma^{\infty}, m, n \in \mathbb{N}^{k}, \sigma^{m}(x)=\sigma^{n}(y)\right\},
$$

where the $Z_{\mathcal{G}_{\Gamma}}\left(U *_{s} V, m\right)$ form a basis of compact open sets for the topology. 
For $m \geq 1$ and $\lambda \in \Lambda_{m}$, let $U_{m, \lambda}:=Z\left(p_{1, m}(\lambda), \ldots, \lambda\right) \subset \Gamma$. So the $U_{m, \lambda}$ are a basis for the topology on $\Gamma=\lim _{\longleftarrow}\left(\Lambda_{i}, p_{i}\right)$. Now for $m \geq 1$ and $\mu, v \in \Lambda_{m}$ with $s(\mu)=s(\nu)$, let

$$
u_{\alpha_{1, m}(r(\mu)) \mu, \alpha_{1, m}(r(v)) v}:=\chi Z\left(U_{m, \mu} *_{s} U_{m, v}, d(\mu)-d(v)\right) \in C_{c}\left(\mathcal{G}_{\Gamma}\right) .
$$

Tedious but routine calculations using the definition of the convolution product and involution on $C_{c}\left(\mathcal{G}_{\Gamma}\right) \subset C^{*}\left(\mathcal{G}_{\Gamma}\right)$ show that

$$
\left\{u_{\alpha_{1, m}(r(\mu)) \mu, \alpha_{1, m}(r(\nu)) v}: m \geq 1, \mu, v \in \Lambda_{m}, s(\mu)=s(v)\right\},
$$

is a Cuntz-Krieger $\left(\lim \left(\Lambda_{n} ; p_{n}\right), \Lambda_{1}^{0}\right)$-family in $C^{*}\left(\mathcal{G}_{\Gamma}\right)$. By the universal property of $C^{*}\left(\lim \left(\Lambda_{n} ; p_{n}\right), \overleftarrow{\Lambda_{1}^{0}}\right)$ (see [1, Section 3]), there is a homomorphism $\psi$ : $C^{*}\left(\underline{\lim }\left(\Lambda_{n} ; p_{n}\right), \Lambda_{1}^{0}\right) \rightarrow C^{*}\left(\mathcal{G}_{\Gamma}\right)$ such that

$$
\psi\left(T_{\alpha_{1, m}(r(\mu)) \mu, \alpha_{1, m}(r(v)) v}\right)=u_{\alpha_{1, m}(r(\mu)) \mu, \alpha_{1, m}(r(v)) v},
$$

for each $m, \mu, v$. The canonical gauge action $\beta: \mathbb{T}^{k} \rightarrow \operatorname{Aut}\left(C^{*}\left(\mathcal{G}_{\Gamma}\right)\right)$ determined by $\beta_{z}(f)(x, m, y):=z^{m} f(x, m, y)$ satisfies $\psi \circ \gamma_{z}=\beta_{z} \circ \psi$ for all $z \in \mathbb{T}^{k}$, where $\gamma$ is the gauge action on $C^{*}\left(\lim \left(\Lambda_{n} ; p_{n}\right), \Lambda_{1}^{0}\right)$. By [21, Proposition 4.3], each $u_{\alpha_{1, m}(r(\mu)) \mu, \alpha_{1, m}(r(\mu)) \mu}$ is nonzero, and it follows from the gauge-invariant uniqueness theorem [1, Theorem 3.5] that $\psi$ is injective. The topology on $\mathcal{G}_{\Gamma}^{(0)}$ is generated by the collection of compact open sets $\left\{U_{m, \lambda}: m \geq 1\right.$, $\left.\lambda \in \Lambda_{m}\right\}$, and the topology on $\mathcal{G}_{\Gamma}$ is generated by the collection of compact open sets $\left\{U_{m, \mu} *_{s} U_{m, v}: m \geq 1, \mu, v \in \Lambda_{m}, s(\mu)=s(\nu)\right\}$. Since $C^{*}\left(\left\{u_{\alpha_{1, m}(r(\mu)) \mu, \alpha_{1, m}(r(v)) v}\right.\right.$ : $\left.\left.m \geq 1, \mu, v \in \Lambda_{m}, s(\mu)=s(\nu)\right\}\right) \subset C^{*}\left(\mathcal{G}_{\Gamma}\right)$ contains the characteristic functions of these sets, it follows that $\psi$ is also onto, and this completes the proof.

REMARK 6.2. The final statement of Proposition 6.1 suggests that we can regard $\lim \left(\Lambda_{i}, p_{i}\right)$ as a skew-product of $\Lambda$ by $G$.

To make this precise, note that for $\lambda \in \Lambda, c(\lambda):=\left(c_{n}(\lambda)\right)_{n=1}^{\infty}$ belongs to $G$, and $c: \Lambda \rightarrow G$ is then a cocycle. There is a natural bijection between the cartesian product $\Lambda \times G$ and the topological $k$-graph $\lim \left(\Lambda_{i}, p_{i}\right)$, so we may view $\Lambda \times G$ as a topological $k$-graph by pulling back the structure maps from $\lim \left(\Lambda_{i}, p_{i}\right)$. What we obtain coincides with the natural definition of the skew-product $\vec{\Lambda} \times{ }_{c} G$.

With this point of view, we can regard Proposition 6.1 as a generalization of [15, Theorem 7.1(ii)] to profinite groups and topological $k$-graphs: $C^{*}\left(\Lambda \times{ }_{c} G\right) \cong$ $C^{*}(\Lambda) \times{ }_{\delta} G$.

EXAMPlE 6.3 (Example 3.3 continued). Resume the notation of Examples 3.3 and 4.2. The resulting projective $\operatorname{limit} \lim \left(\Lambda_{n}, p_{n}\right)$ is the topological 1-graph $E$ associated to the odometer action of $\mathbb{Z}$ on the Cantor set as in [21, Example 2.5(3)]. That is, $E$ can be realized as the skew-product of $B_{1}^{*}$ by the 2 -adic integers $\mathbb{Z}_{2}$ with respect to the functor $c: B_{1}^{*} \rightarrow \mathbb{Z}_{2}$ determined by $c(f)=(1,1,1, \ldots)$, where $f$ is the loop edge generating $B_{1}^{*}$. 


\section{References}

[1] S. Allen, 'A gauge invariant uniqueness theorem for corners of higher rank graph algebras', Rocky Mountain J. Math. 38 (2008), 1887-1907.

[2] T. Bates, J. Hong, I. Raeburn and W. Szymański, 'The ideal structure of the $C^{*}$-algebras of infinite graphs', Illinois J. Math. 46 (2002), 1159-1176.

[3] D. Drinen and M. Tomforde, 'The $C^{*}$-algebras of arbitrary graphs', Rocky Mountain J. Math. 35 (2005), 105-135.

[4] S. Echterhoff, S. Kaliszewski, J. Quigg and I. Raeburn, 'A categorical approach to imprimitivity theorems for $C^{*}$-dynamical systems', Mem. Amer. Math. Soc. 180 (2006), viii+169.

[5] M. Enomoto and Y. Watatani, 'A graph theory for $C^{*}$-algebras', Math. Japon. 25 (1980), 435-442.

[6] P. A. Fillmore, A User's Guide to Operator Algebras, Canadian Mathematical Society Series of Monographs and Advanced Texts (John Wiley \& Sons, New York, 1996), pp. xiv+223.

[7] S. Kaliszewski and J. Quigg, 'Mansfield's imprimitivity theorem for full crossed products', Trans. Amer. Math. Soc. 357 (2005), 2021-2042.

[8] — 'Landstad's characterisation for full crossed-products', New York J. Math. 13 (2007), 1-10.

[9] A. Kumjian and D. Pask, 'Higher rank graph $C^{*}$-algebras', New York J. Math. 6 (2000), 1-20.

[10] A. Kumjian, D. Pask and I. Raeburn, 'Cuntz-Krieger algebras of directed graphs', Pacific J. Math. 184 (1998), 161-174.

[11] A. Kumjian, D. Pask, I. Raeburn and J. Renault, 'Graphs, groupoids and Cuntz-Krieger algebras', J. Funct. Anal. 144 (1997), 505-541.

[12] A. Kumjian, D. Pask and A. Sims, ' $C$ *-algebras associated to coverings of $k$-graphs', Documenta Math. 13 (2008), 161-205.

[13] M. B. Landstad, 'Duality for dual $C^{*}$-covariance algebras over compact groups', Preprint, 1978.

[14] _ 'Duality theory for covariant systems", Trans. Amer. Math. Soc. 248 (1979), 223-267.

[15] D. Pask, J. Quigg and I. Raeburn, 'Coverings of k-graphs', J. Algebra 289 (2005), 161-191.

[16] D. Pask, I. Raeburn, M. Rørdam and A. Sims, 'Rank-2 graphs whose $C^{*}$-algebras are direct limits of circle algebras', J. Funct. Anal. 239 (2006), 137-178.

[17] J. Quigg, 'Landstad duality for $C^{*}$-coactions', Math. Scand. 71 (1992), 277-294.

[18] D. I. Robertson and A. Sims, 'Simplicity of $C^{*}$-algebras associated to higher-rank graphs', Bull. London Math. Soc. 39 (2007), 337-344.

[19] G. Robertson and T. Steger, 'Affine buildings, tiling systems and higher rank Cuntz-Krieger algebras', J. Reine Angew. Math. 513 (1999), 115-144.

[20] T. Yeend, 'Topological higher-rank graphs and the $C^{*}$-algebras of topological 1-graphs', Contemp. Math. 414 (2006), 231-244.

[21] _ ' 'Groupoid models for the $C^{*}$-algebras of topological higher-rank graphs', J. Operator Theory 57 (2007), 95-120.

DAVID PASK, School of Mathematics and Applied Statistics, University of Wollongong, NSW, 2522, Australia e-mail: dpask@uow.edu.au

JOHN QUIGG, Department of Mathematics and Statistics, Arizona State University, Tempe, Arizona, 85287, USA

e-mail: quigg@asu.edu

AIDAN SIMS, School of Mathematics and Applied Statistics, University of Wollongong, NSW, 2522, Australia

e-mail: asims@uow.edu.au 